\title{
CONNECTIVE TISSUE MASSAGE
}

\author{
By MARIA EBNER, M.C.S.P.
}

(Teacher's Certificate).

\author{
Diploma in Physical Education (Vienna University), former Principal of School and Department of Physiotherapy of the \\ United Leeds Hospital, England.
}

Connective Tissue Massage represents a type of Physiotherapy Treatment of which I became aware soon after the second world war when the Chartered Society of Physiotherapy inyited a number of German Physiotherapists to its Annual Congress in London. One of these Physiotherapists followed my invitation to Leeds where he demonstrated the technique on a member of my staff who suffered from severe and very acute "fibrositis" of the neck. The result of two treatments was amazing in giving almost immediate relief of a condition which had existed for a long time and had been treated by various forms of conventional physiotherapy. Some of the events during the treatment such as the general feeling of warmth arising in a fairly cold room were to say the least of it, puzzling. My interest in this form of treatment was very much aroused, but the difficult conditions of organising a big department and running a school prevalent in the years after the war, prevented me from going to Germany and study the method. This was at last possible in 1954. Since then I have not only learned to appreciate the value of this form of treatment in a variety of conditions for which there was little other help available but it has contributed to the development of a different outlook on "therapy" in myself. The unsatisfactory assessment of a patient as a "shoulder-syndrome" or a case of "vascular insufficiency" and many other similar descriptions of patient's symptoms, has given place to an appreciation of a patient where certain symptoms are only the obvious signs of a dysfunction of the whole organism. On my return from Germany I treated personally or through members of my staff under my supervision several hundred patients with very satisfactory and sometimes amazing results. In the beginning the patients referred by consultants were usually patients in which more conventional forms of therapy had failed, later on patients were selected, taking into account the results previously achieved. A second visit to Germany in 1958, this time to Frieburg im Breisgau, produced some new aspects of this form of therapy.

I mentioned previously my changing attitude in the physiotherapeutic approach to the patient and should like to enlarge on this statement. Many of the patients to be found in Physiotherapy Departments are suffering from a variety of "soft tissue lesions". Two components are therefore always involved even if the condition is originally due to injury of boney or nervous tissue. The two components involved are the connective tissue and the circulatory system. The circulatory system in its widest sense consists of a system of tubes dealing with an amount of fluid, the quantity of which only alters within small limits, provided the heart and kidneys are working normally. Accumulation of fluid, due to inflammatory processes anywhere in the body or lack of fluid due to interference with the vascular nerve supply, must therefore cause alterations in the fluid contents of the tissues in other parts. The tissue which is primarily affected by alterations in fluid contents is connective tissue. Lack of fluid or alterations in the consistency of the fluid will not only affect the consistency of connective tissue by also its extensibility. Connective tissue forms the body surface and also forms an integral part of all body structures. It is continuous throughout the body. Alterations in its tension anywhere in the body must therefore be reflected in other parts of the body and interfere with function. Loose connectiye tissue almost assumes the function of an organ of metabolism, acid-base equilibrium, water and salt balance, osmotic pressure regulation all take place through the medium of connective tissue. Sometimes there is active participation of connective tissue cells in these processes. Circulation plays a decisive part in the formation of connective tissue. Circulation is partly under nervous control (as mentioned) partly under chemical control such as endocrine secretions and release of substances such as histamine and heparin from the Mast cells which are one of the cellular elements of connective tissue.

These thoughts offer an explanation why we find extensive connective tissue changes in a variety of conditions which are not associated with injury such as states of mental tension in many people who have lost the art of relaxation. Results of this tension such as frequent headaches, backache, shoulder-syndrome are often only treated with drug therapy and therefore the changes which occur as a result of circulatory changes in the connective tissue are not cleared up. The pain and functional impairment due to these tissue changes form part of a vicious circle increasing the mental tension. These connective tissue changes can often be observed in the body conture in the back. (See literature.) It is therefore essential to consider the patient as a whole, normalize the connective tissue tension over the whole body by influencing the circulation on the body surface. This can be achieved by connective tissue massage in the back. Widespread connections along circulatory pathways and segmental nerve supply will influence the circulation not only over the body surface but in somatic and visceral structures associated with the areas under treatment. Almost all treatments start in the sacral area for various reasons. The sacral area gives rise to part of the parasympathetic outflow and the connective tissue covering it is continuous with the connective tissue covering the upper part of the trunk and arms and with the connective tissue covering the buttocks and the legs. Empirically it has been found that this increase in peripheral circulation promotes a condition of general relaxation and a feeling of wellbeing. Patients sleep better which helps to break the vicious circle of tension buildup.

In order to make this article more useful, I am not generally referring to facts which have been discussed in detail in the book "Connective Tissue Massage" but I am trying to concentrate on conditions present in patients who have been presented to me during the courses which I have been able to hold in South Africa. I have been particularly impressed by the prevalence and frequency of peripheral vascular lesions both in Whites and non-Whites for which I could not obtain an explanation related to either climatic or nutritional factors. I should be very grateful if any research or literature existing on this subject could be brought to my notice. Since the publication of the book on this subject $I$ have had further opportunity to treat patients 
suffering from the conditions mentioned and $I$ therefore proposed to discuss in more detail than was possible previously, migraine and intermittent claudication.

\section{Migraine}

The diagnosis of migraine is often applied to various forms of headache, which need not necessarily produce the more specific symptoms of true migraine, i.e. visual disturbances and/or nausea. All forms of headache benefit from the application of C.T. massage but as headache is a symptom which be due to a variety of causes, it will lead to a more successful approach if some of these causes and their symptoms will be discussed.

Causes: Headache may be a symptom in post-concussional syndrome and during the inspection of the back the zones between the scapulae, along the cervical spine and along the occipital curved line will be particularly pronounced. Changes may be palpated along the border of the upper fibres of trapezius and along the borders of sterno-mastoid.

In the presence of generalized rheumatism, headaches may be a troublesome additional symptom and are often associated with paraesthesia in the upper limbs particularly noticeable in the morning on waking up. The main headache zone between the scapulae will again be present but additional pronounced zones may be noticed over the posterior aspect of deltoid. Headaches with additional symptoms of nausea which often may lead to vomiting will of ten produce distinctly visible and palpable liver or intestinal zones in addition to the always-present interscapular zone. In these cases it will be useful to direct some investigation and the patient's attention to the occasions when these headaches appear. It will be possible to find frequently an association between headache periods and some food allergy.

In other patients it may be possible to detect an association between headache periods and the menstrual cycle. In these patients the lower headache zones (see book chart) will be frequently found.

In patients suffering from a condition which is often diagnosed as tension headaches all the upper headache zones will be very pronounced but the whole back may show conture alterations with special liver or intestinal zones present.

The above remarks will make it clear how important the first investigation is in order to assess the patient's condition. It is often helpful if the patient has a headache when they come for their first treatment. Visual investigation according to the back-chart will reveal the presence of headache zones and the additional presence of e.g. liver zones or menstrual zones or special zones of tension in the cervical region may provide a guide to the underlying cause of the headaches. Manual investigation will reveal changes in tissue consistency or tissue sensitivity in certain areas. All these findings will help to plan the treatment more intelligently. It is most important to have an open mind and not expect "typical" symptoms in patients who suffer from headaches. Some patients who suffer from severe migraine attacks with continuous vomiting may be perfectly well the next day and other may feel off colour for several days. It is always important to treat thoroughly the sacral area, as in all types of headache there seems to exist a relationship between the sacral area and the head. For the rest of the treatment the importance of emphasis on other areas varies. A general easing of the condition can almost invariably be observed after 1-3 treatments of the sacral area in all patients. In some patients the final improvement occurs when the latissimus dorsi and the area between the scapulae is treated, in others the final clearance occurs when treatment is given in the cervical region and along the borders of trapezius. In other patients the liver zone represents the crucial treatment area.
To give a general guide I shall attempt to discuss these patients falling into three main groups. I should however like to stress again that a clear division is often not possible and various underlying causes may combine to give the patient's symptoms.

\section{Headaches after Trauma}

As far as possible the treatment should be carried out in sitting and concentrated on the basic section for 2-3 sessions with the addition of the latissimus dorsi border. Further progression towards the cranium will have to be guided by the patient's condition. The patient should however not be discharged until the occipital curved line shows normal tension and absence of special pain sensitivity.

\section{Headaches Associated with Menstrual or Digestive Disturbances}

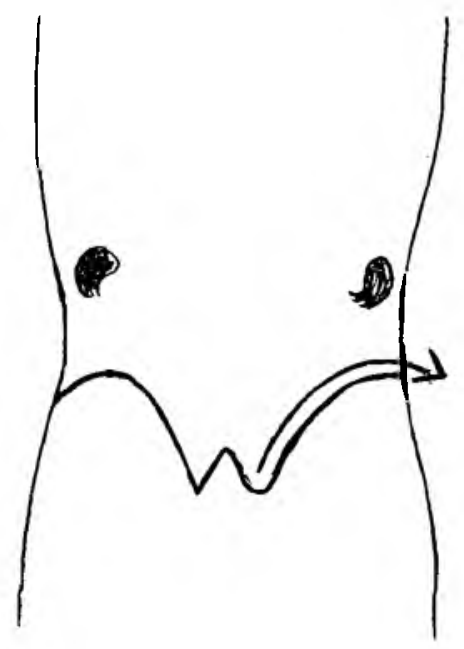

LIVER-STROKE

The patient should be in lying and the Physiotherapist should be sitting on the right side of the patient. The stroke can be carried out with the right or the left hand, at first with very little depth, gradually increasing in depth.

These patients usually show a distinct headache zone between the scapulae and additional zones over the liver area and the sacrum. For a number of treatments it is essential to concentrate on the basic section, carrying out the treatment in sitting. Long strokes will not be possible in many cases as the patient's response is often only a "tickling" sensation in response to the stroke instead of the scratching or cutting sensation. Short strokes must therefore only be applied until the response alters. It may often be necessary to include the great trochanter area in the early treatments. This treatment is best carried out in half lying or lying. As soon as the progress of the treatment permits the liver area must be included in the treatment, concentrating at first with short strokes on the angle between the last rib and the vertebral column, including latissimus dorsi into the treatment and gradually attempting to apply the subcostal stroke with the patient in the lying position.

This "liverstroke" must be applied with no depth in the beginning as the area is often very sensitive. The feeling of nausea or sickness may often be abolished with this stroke. 
If the headaches are definitely associated with the menstrual cycle, treatment as described in "Connective Tissue Massage" must be applied.

\section{Headaches associated with Hypertension}

Back investigation in these patients often produces acute cutting sensation in the sacral areas, distinct zones between the scapulae and very much increased sensitivity to palpation in the whole cervical and shoulder areas up to the occipital curved line. Early treatments must be concentrated on the basic section and the latissimus dorsi, carried out in sitting if possible. Gradual progression must include the whole back and cervical region, scapulae and clavicles. It is very important to abandon the treatment of the paravertebral thoracic area until a clear cutting sensation can be obtained. Frequent strokes over the clavicles and anterior release strokes are very important as the autonomic balance in these patients is often very labile. It is also important that these patients rest for 20-30 minutes after the treatment. To teach general relaxation to these patients proves often of additional benefit.

In order to illustrate some of the points, previously discussed, the following case history may prove useful.

Patient: Female, aged 55. Occupation: Housewife.

$7 / 7 / 1962$. This patient's case history revealed that migraines started in 1948 after her husband's death, having one or two attacks per week. (There is a family history of migrainegrandmother and niece suffer from it). The number of attacks has decreased during the last two years. At present she has one at intervals of approximately 10 days. She sleeps badly and usually gets up during the night to make some tea. She aIways wakes with a headache which usually lasts until midday. When she has a migraine attack, she usually wakes at about 6 a.m. The headache increases in intensity for approximately 3-4 hours when she starts attacks of vomiting. This stage passes after another 3-4 hours, when the patient is able to go to sleep for 3-4 hours with the help of "migral". The pain usually starts in the frontal area and passes later to the back of the skull. She avoids long car journeys or visits to the city as it brings on an attack of migraine. She cannot tolerate bright light or sitting in the sun.

\section{Treatment:}

1. Basic Section: The back was very tense and all strokes very cutting.

2. Back to $\mathrm{C} 7$ and anterior pelvic strokes.

3. The patient reported that the morning headaches cleared up much more quickly. Back treated to $\mathrm{C} 1$.

4. Patient arrived with ordinary headache. Back treated to $\mathrm{Cl}$, Latissimus dorsi, clavicles and trapezius. The pain improved after the ribstrokes and completely disappeared after the treatment of Latissimus dorsi.

5. Two days ago the patient woke with migraine, but less severe than before and did not vomit. The migraine lasted until 6 p.m. On arrival the patient reported that for the first time she did not wake with a headache today. Treatment as last time. The patient is now sleeping well and has not found it necessary to get up during the night to make tea since the second treatment.

6. The patient reported that she had been to the city until 4 p.m., the first time for many years and had no headache in the evening.

7. On arrival the patient reported that she had a slight migraine, suffered from nausea, but had not been sick, Treatment as before. The feeling of nausea cleared after the rib-strokes and the headache completely disappeared after the anterior pelvic strokes.
Six further treatments were given at the rate of three times per week. The patient reported that she had been headachefree for one week, could work in the garden, tolerate bright light and could sit in the garden in the sun.

The patient continued treatment at the same rate and had only short episodes of ordinary slight headaches.

$24 / 8 / 1962$. The patient reported that she had been motoring all day on the previous day and felt delighted as she had not risked such a journey for many years. She returned without headache.

The patient continued with treatment three times per week until the end of September 1962. She had now resumed an ordinary social life, went to the theatre, had visitors and worked with the "Women's Guild" without feeling any ill-effects.

Two further treatments were given, one in October and one in November. The patient reported that "she felt like a new woman, 10 years younger". She had no ordinary headaches or migraines during these two months and discontinued treatment in December 1962. She reported a year later that she was still feeling very well.

\section{Intermittent Claudication}

This condition has been previously discussed (Ebner C.T.M., pages 150-154). Further experience has shown that the effect of treatment can often be enhanced if the patients are encouraged to rest in the recumbent position for half an hour to one hour after treatment. Where possible the treatment should therefore be carried out in the patient's home or arrangements made that the patient can have undisturbed rest in the department before embarking on the homeward journey. It is claimed by German investigators that the autonomic tone swings over to the parasympathetic side after C.T. massage which proves beneficial to these patients, who very often are tense and find it difficult to relax. The condition of these patients is often aggravated by additional pathology affecting other organs. Gastric or duodenal ulcers or heart conditions may be present and the rest period in all these cases is therefore very important, in order to allow the circulatory system to adapt itself to the altered situation produced by the C.T. massage treatment. It is also often important to teach these patients general relaxation and draw their attention to the fact that a more tolerant approach to difficulties arising in daily life would help their condition. Many of these patients are used to managing a great deal of work in their life and have therefore adopted very quick walking habits. It is often helpful to advise them to walk a little bit more slowly and avoid a situation where they have to take definite rest periods. In some cases additional benefit can be derived if the back is treated to the level of T7 and special attention is given to the liver zone. Should the patient suffer additionally from headaches, the treatment must be gradually extended over the whole back. Fairly long intervals without treatment are possible once the condition has resolved itself to the extent that the patient can follow his ordinary working routine at a slightly reduced speed. This may demand initially 20-30 treatment sessions and it is advisable in many cases to repeat yearly a short course of treatment.

The following case history may illustrate some of the points made:

Patient: Male. Age, 60 years. Diagnosis: Hardening of arteries, intermittent claudication.

19/4/1964. On investigation the patient reported that he had felt some leg pain after walking longer distances but the condition had become much aggravated after return from a pilgrimage when he had been on his feet for many hours without rest. At present pain occurs after very short distance walking-he has to slow down but does not have 
to stop completely. There is no pain after a night's rest but it soon returns after resuming the tasks of a daily routine which involved a great deal of walking and standing. The patient's normal walking pace was very quick.

There was a positive arterial zone over both buttocks, more pronounced on the left.

Treatment:

1. Forward lying basic section, two thighs as far as the popliteal space, including the two heads of gastrocnemeus. A slight cutting reaction over the buttocks could only be obtained after stimulation of the trigonum lumborum. First stroke reaction was only "tickling". After the treatment the patient reported that he felt fine and the legs much more alive.

2. Patient reported that he felt fine and had mowed the lawn for $1 \frac{3}{4}$ hours without ill effects.

\section{Treatment:}

Basic section and both legs including the feet. The stroke reaction was much sharper except over the feet which only gave a "tickling" reaction. The lower legs were only treated with short strokes. General relaxation taught.

3. Patient reported he was walking with much greater ease. The same areas were treated. A much better erythema was obtained, the short strokes along the medial and lateral border of the feet were felt as very sharp.

4. Felt very well after the last treatment for two days when some discomfort was felt in the right ilio-tibial tract and the right calf. Treatment of back extended to $\mathrm{T} 7$ in the forward lying position. The two legs were treated in the lying position and treatment specially concentrated with short strokes on the ilio-tibial tracts and the popliteal space.

These four treatments were carried out at 3-4 days interval. A necessary journey caused interruption in the course of treatment for one month.

5. The patient could carry out all necessary walking without having to stop, discomfort in the calves, however, after quick walking necessitated sometimes slowing down of pace.

\section{Treatment:}

Back to T7, both lower extremities. Cutting reaction obtained everywhere.

6. Patient reports that he can do everything, only slight discomfort in calves on quick walking.

Fourteen further treatments were given at weekly intervals. The walking pace could gradually be increased. The patient went for a three-week holiday. During the holiday he had a slight flareup of his gastric ulcer. After long walks he felt tired but had no acute pain.

26/11/1964. The treatment was resumed. Areas treated were the back to T7, latissimuus dorsi and both legs including the feet. After the first treatment the gastric ulcer gave no further trouble. Seven more treatments were given to the same areas at weekly intervals. The patient only experienced discomfort in the calves on quick uphill walking and the left hallux felt less alive than the rest of the foot. During this period the patient had a great deal of work which involved walking and standing and this could be carried out without discomfort.

After my return from South Africa I saw the patient again on April 21st 1965. During the period of $3 \frac{1}{2}$ months without treatment the patient had no ulcer trouble and no leg discomfort except on quick uphill walking.

Literature: Connective Tissue Massage, Theory and Therapeutic Application. Maria Ebner, 1962. E. and S. Livingstone Ltd., Edinburgh.

\section{SOFT TISSUE INJURIES OF ANKLE AND CALF}

\author{
By MARIA EBNER, M.C.S.P.
}

(Teacher's Certificate).

(Lecture-Demonstration given to Physiotherapy Students)

\begin{abstract}
These injuries are often considered trivial but may incapacitate the patient for a long time. If the treatment is not planned on physiological lines, these injuries may recur again at frequent intervals and eventually force the patient to discontinue with athletic activities. I have chosen for illustration of the underlying principles of treatment two frequently-occurring injuries, i.e., "sprained ankle" and
\end{abstract} "partial tear of gastrocnemeus fibres".

The cause of these injuries is always the use of tissue beyond physiological limits, resulting in a tear or stretch of formed or unformed connective tissue. As a result of the tear, blood vessels are injured, haemorrhage occurs, exudation of tissue fluid takes place and a haematoma forms. The haematoma gradually becomes organised into scar tissue which has a tendency to contract and shorten, very often including some nerve fibres into the scar tissue. If the scar tissue is part of a muscular structure a different coefficient of expansion is present between the muscle fibres and the scar tissue. Future functional use of the muscle will often cause pain due to the caught nerve fibres and future tears will often result due to the different coefficient of expansion between the two tissues.

A physiological approach to these problems will have to follow the principles of tissue repair and assist them with physiotherapeutic measures as far as possible.

The first principle of treatment in these injuries is therefore to limit the amount of haemorrhage and exudation and encourage absorption as far as possible in order to limit the amount of unavoidable scar tissue. Static hyperaemia as a result of the injury must therefore be changed into active hyperaemia so that inflammatory products can be carried away in the blood and lymphstream.

The second principle of treatment applies to the normal use of the part within physiological limits in order to avoid alienation occuring in the cortical pathway.

The two examples chosen may help to illustrate the abovementioned principles.

\section{Sprained Ankle}

It is commonly taught that a sprained ankle is a lesion of the lateral.ligament of the ankle joint due to an inversion strain. This explanation assumes that a ligament is the first line of defence if unphysiological force is applied to a joint. This is incorrect. Ligaments as well as muscles possess as part of their structure stretch receptors which record applied stretching strains beyond their physiological stretching ability. As a result of this stretch stimulus muscles reflexly contract to protect structures such as capsules and ligaments which have very little extensibility. If the applied force is too great, inextensible structures such as the periosteal attachments of muscles or fibres at the junction of tendon and muscle or ligaments passing over a joint or forming part of the joint capsule, will tear. If the inversion strain is too suddenly or too forcefully applied and injury occurs, it is therefore reasonable to look for injury in the above-mentioned structures. The muscles affected in an inversion strain which is not strong enough to produce a fracture will be the evertors, i.e., peroneus longus, peroneus brevis and peroneus tertius. If it is accepted that the weakness and pain after a sprained ankle is due to a muscle lesion rather than a ligamentous lesion the common sites of pain and swelling can easily be explained.

Pain is often present at the base of the fifth metatarsal which represents the insertion of peroneus brevis which is torn off partially from its insertion. Pain and swelling in front of the lateral malleolus represents an injury to veroneus 